\title{
Checkpoint inhibitors in melanoma and early phase development in solid tumors: what's the future?
}

\author{
Paolo A. Ascierto ${ }^{1 *}$ and Grant A. McArthur ${ }^{2,3^{*}}$
}

\begin{abstract}
Anti-programmed death (PD)-1 and PD-ligand (L)-1 checkpoint inhibitors have revolutionized the therapy of several cancers. Immunotherapy of cancer can offer long-term durable benefit to patients, is active regardless of tumour histology, has a unique immune-related safety profile, and can be used in combination with other cancer treatments. In addition, recent research has shown that immune-based therapy can be used as adjuvant therapy, that outcomes may be influenced by dose, and that clinical activity is observed in patients with brain metastases. Despite our increased understanding of these agents, there are still several important questions that need to be answered. These include strategies to overcome primary and acquired resistance, the influence of mutational status on treatment outcomes, the optimal duration of treatment, and the need to identify novel combination regimens that offer increased anti-tumour potency and/or reduced toxicity. Here we review recent developments in these areas, with particular focus on new data reported at the 2017 ASCO Annual Meeting.
\end{abstract}

Keywords: Anti-PD-1, Anti-PD-L1, Combination therapy, Immunotherapy, Melanoma

\section{Background}

Anti-programmed death (PD)-1 and PD-ligand (L)-1 checkpoint inhibitors have revolutionized the therapy of several cancers, initially in advanced melanoma but now in several other tumour types, including non-small-cell lung cancer (NSCLC), squamous cell carcinoma of the head and neck (SCCHN), urothelial bladder cancer, renal cell cancer and Hodgkin's lymphoma.

In the past few years, we have learned that immunooncology (I-O) therapy can offer long-term benefit to patients, is active regardless of tumour histology, has a unique safety profile, and can be used in combination with other cancer treatments (e.g. chemotherapy, targeted therapy, radiation). Most recently, research has shown that I-O therapy can be used as adjuvant therapy [1], outcomes may be influenced by dose [2], and that

\footnotetext{
*Correspondence: paolo.ascierto@gmail.com;

p.ascierto@istitutotumori.na.it; grant.mcarthur@petermac.org

${ }^{1}$ Istituto Nazionale Tumori IRCCS Fondazione "G. Pascale", Via Mariano

Semmola, 80131 Naples, Italy

2 Peter MacCallum Cancer Centre, Melbourne, VIC, Australia

Full list of author information is available at the end of the article
}

clinical activity is observed in patients with brain metastases [3].

However, there are still several important questions that need to be answered. These include strategies to overcome primary and acquired resistance, the influence of mutational status on treatment outcomes, the optimal duration of treatment, and the need to identify novel combination regimens that offer increased anti-tumour potency and/or reduced toxicity. Here we review recent developments in these areas, with particular focus on new data reported at the 2017 ASCO Annual Meeting.

\section{Treatment resistance}

Although anti-PD-1/PD-L-1 therapy has improved clinical outcomes, the majority of patients still fail to respond, due to intrinsic resistance as determined by standard oncology response criteria [4]. Also, among those patients who do respond, disease progression can occur due to acquired resistance [5]. Very little data from humans actually exists and some data comes from preclinical models. However, previous reports in humans have indicated that the most important mechanisms of 
primary resistance might include the loss of major histocompatibility complex (MHC), an increase of the number of regulatory cells into the tumour microenvironment (e.g. regulatory $\mathrm{T}$ cells [Tregs], myeloid derived suppressor cells [MDSCs], tumour-associated macrophages [TAMs], etc.), an increase of the production of immunosuppressive cytokines (such as interleukin [IL]-10 and TGF- $\beta$ ), and the upregulation of checkpoint molecules (such as LAG3). Recently, biopsy samples from four patients with metastatic melanoma who had had an initial response to anti-PD-1 therapy with pembrolizumab followed by disease progression suggested that mutations in genes encoding for interferon receptor-associated Janus kinase (JAK) 1, JAK2 or $\beta 2$-microglobulin (a necessary constituent of the MHC class I complex) may be involved in the development of acquired resistance [6]. Similar mechanisms have also been described for resistance to anti-CTLA-4 [7].

\section{Mutational status}

Immuno-oncology can be effective regardless of tumour histology and mutational status. Data from the Italian ipilimumab expanded access programme clearly showed no difference in term of overall survival (OS) between patients who harbored the BRAF mutation and those who were BRAF wild-type [8]. However, results from two recent clinical trials, the CA184-169 study of ipilimumab $10 \mathrm{mg} / \mathrm{kg}$ versus ipilimumab $3 \mathrm{mg} / \mathrm{kg}$ [2] and the CheckMate 067 trial of ipilimumab plus nivolumab versus ipilimumab or nivolumab monotherapy [9], showed better outcomes with, respectively, ipilimumab $10 \mathrm{mg} /$ $\mathrm{kg}$ and the combined ipilimumab plus nivolumab regimen in BRAF-mutated patients. Whilst it is possible that could simply be attributed to the limitations of subgroup analyses with imbalance between the groups, we speculate that new biological data in NSCLC coupled with recently published data from melanoma provide a plausible explanation.

Second-line treatment with anti-PD-1/PD-L1 therapy is ineffective in patients with EGFR mutations in NSCLC [10-12]. The EGFR mutation is responsible for low interferon (IFN) $-\gamma$ signature and higher expression of CD73, which metabolisises the conversion of AMP to adenosine [13]. Adenosine is highly immunosuppressive with several effects on immune cells and the tumor microenvironment. Streicher et al. reported that median CD73 expression was increased 10 fold compared to wild-type cell lines in EGFR-mutant NSCLC cell lines, while anti-EGFR tyrosine kinase inhibitor treatment resulted in dose-dependent inhibition of CD73 expression, suggesting a causal relationship between the EGFR pathway and CD73 expression [14]. In addition, EGFRmutant tumours had $\geq 2$ fold increased expression of
CD73 compared to wild-type in NSCLC adenocarcinoma patients. These EGFR mutants had significantly lower levels of a IFN- $\gamma$ signature, previously reported to be associated with enhanced benefit from the anti-PDL1 agent, durvalumab [13]. High CD73 expression is also associated with low PD-L1 expression [15].

A recently published study from Young and colleagues indicates BRAF mutation may also create CD73-dependent immune suppression in melanoma [16]. These investigators found a possible association between higher expression of CD73 and BRAF mutation. They were also able to demonstrate enhanced efficacy in preclinical in vivo models when adenosine signalling was inhibited in addition to combined BRAF and MEK inhibition. Collectively these results suggest that over-expression of CD73 in EGFR-mutant NSCLC patients and BRAFmutant melanoma may, in part, explain the reduced benefits from anti-PD-1/PD-L1 therapy in EGFR-mutant NSCLC and the benefits from higher doses of ipilimumab or the combination of ipilimumab and nivolumab in BRAF-mutant melanoma (Fig. 1). These data set the scene for evaluating inhibition of CD73 with anti-PD-1/ PD-L1 therapy in patients with oncogene driven cancers.

\section{Duration of treatment}

Currently, the optimal duration of therapy with antiPD-1/PD-L1 agents remains unknown. In melanoma the standard of care has switched from ipilimumab (with which only four cycles of treatment can provide a longterm benefit of 10 years for $20 \%$ of patients) [17] to anti PD-1/PD-L1 therapy, which is recommended until disease progression or occurrence of toxicity. However, it is not know if a shorter duration of PD-1/PD-L1 therapy might be equally effective. In 2016, data from a cohort of 61 patients in complete remission who stopped treatment with pembrolizumab at a median of almost 2 years showed that, at a median follow-up of about 10 months from stopping pembrolizumab therapy, only two patients had disease recurrence [18].

Robert et al. reported data from the KEYNOTE-006 study that showed a durable response [19]. At a median follow-up of nearly 3 years, 33-month OS with pembrolizumab compared to ipilimumab was $50 \%$ versus $31 \%$ and PFS was $39 \%$ versus $14 \%$. In 104 patients who stopped pembrolizumab treatment after 2 years as per the study protocol, the estimated PFS was $91 \%$ at a median follow-up of 9.7 months after completing 2 years of pembrolizumab. Importantly, it was not just patients with complete responses who maintained PFS after stopping therapy, with a PFS rate of $91 \%$ in patients with partial responses and $83 \%$ in patients with stable disease (compared with $95 \%$ of patients with complete responses). This finding may be important in helping define the 


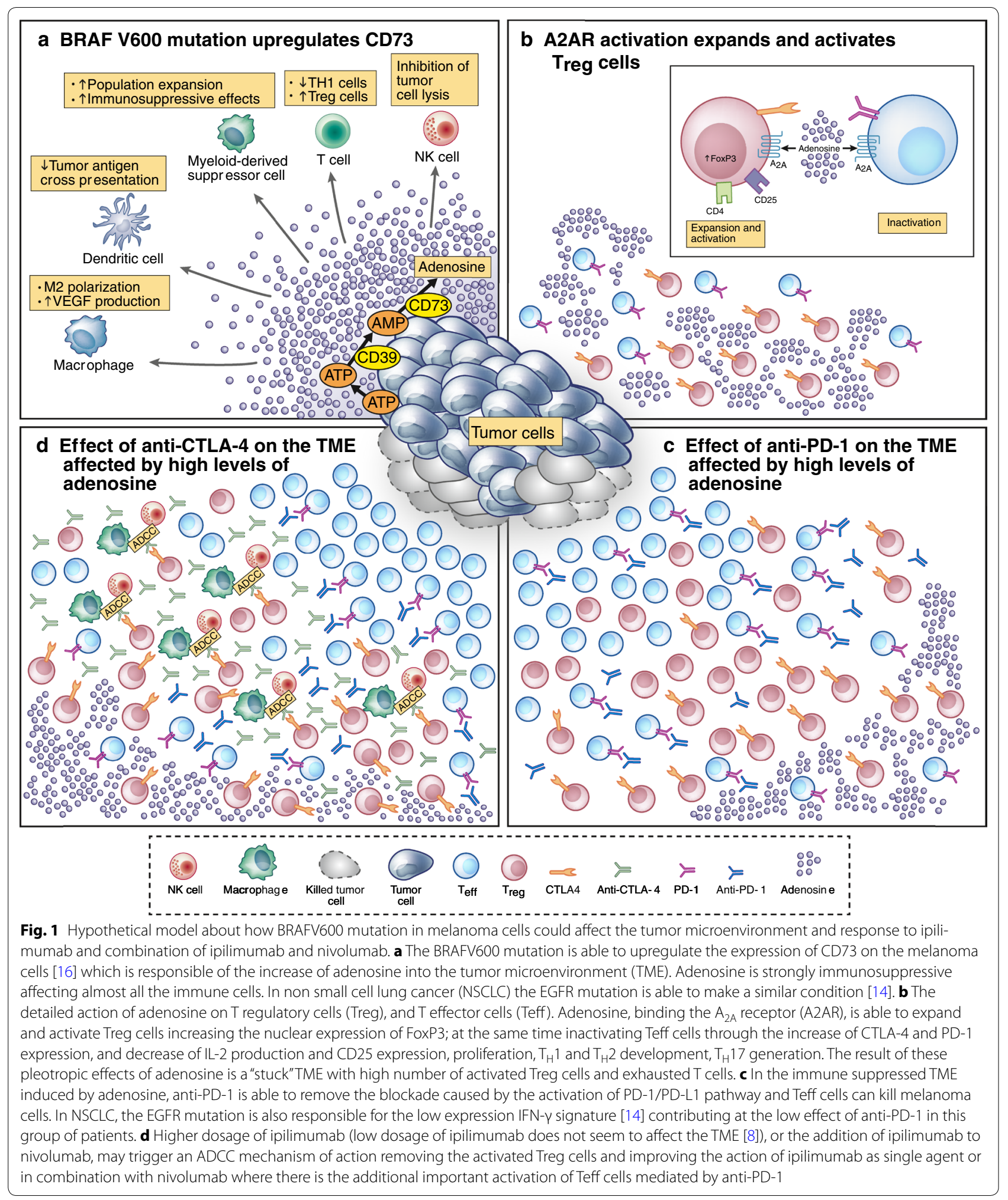

duration of therapy that is needed, with the possibility of shorter treatment and the associated benefits for patients as well as reduced healthcare costs.

\section{Brain metastases}

Melanoma brain metastases are a common clinical presentation associated with poor prognosis. Surgery and/ 
or stereotactic radiation therapy are widely used for oligometastatic disease and whole brain radiation therapy is used for extensive or leptomeningeal disease. However, these modalities have not been shown to improve survival and cannot impact extracranial disease and are associated with significant early and late neurotoxicity.

Experience with immunotherapy for patients with melanoma brain metastases is limited but two ongoing studies were reported at ASCO 2017. In the phase II CheckMate 204 study, nivolumab plus ipilimumab had a high intracranial ORR of $55 \%$ (21\% complete responses) at a median follow-up of 9.2 months in 75 patients [20]. Median duration of response was not reached. Sixmonth PFS was $67 \%$ with median PFS was not reached. The safety profile of the combination was consistent with that in patents without brain metastases, with no unexpected neurologic safety signals. As in patients without brain metastases the benefits of the combination of ipilimumab and nivolumab need to be balanced with the greater toxicity when being compared to monotherapy with nivolumab. Similarly, in the phase II ABC trial in 76 patients with melanoma brain metastases and no previous checkpoint inhibitor treatment, intracranial ORR was $42 \%$ with combined ipiliumumab plus nivolumab versus $20 \%$ with nivolumab alone [21]. Median PFS with the combination was 4.8 months with 6-month PFS of $46 \%$. Median duration of response was not yet reached. In drug treatment-naïve patients, intracranial ORR was 50\% with combined treatment ( $21 \%$ with nivolumab alone). Intracranial ORR was $16 \%$ in patients previously-treated with BRAF/MEK inhibitors. Patients with symptomatic or leptomeningeal metastases or who had previous local therapy responded poorly to nivolumab alone.

Other studies in patients with melanoma brain metastases have shown clinical activity with targeted therapies. In the COMBI-MB trial, dabrafenib plus trametinib in 125 patients with BRAF V600-mutant melanoma brain metastases had a promising intracranial response rate of $58 \%$ and median duration of intracranial response of 6.5 months [22]. It is possible that targeted therapy may result in more responses but the duration of response may be lower when compared with I-O (Fig. 2).

\section{Novel combinations}

The potential of anti-PD-1s in combination with other novel agents is also being explored. Figure 3 summarizes the proposed mechanism of action of the recent emergent compounds. The impetus behind the development of novel combinations is to identify regimens that can overcome primary or acquired resistance to anti-PD-1/PD-L1 and/or reduce toxicity compared to combination therapy with anti-CTLA-4 and anti-PD-1. Two studies reported appeared to confirm a potential role for the indoleamine-pyrrole 2,3-dioxygenase (IDO) inhibitor epacadostat in combination with an antiPD-1 agent.

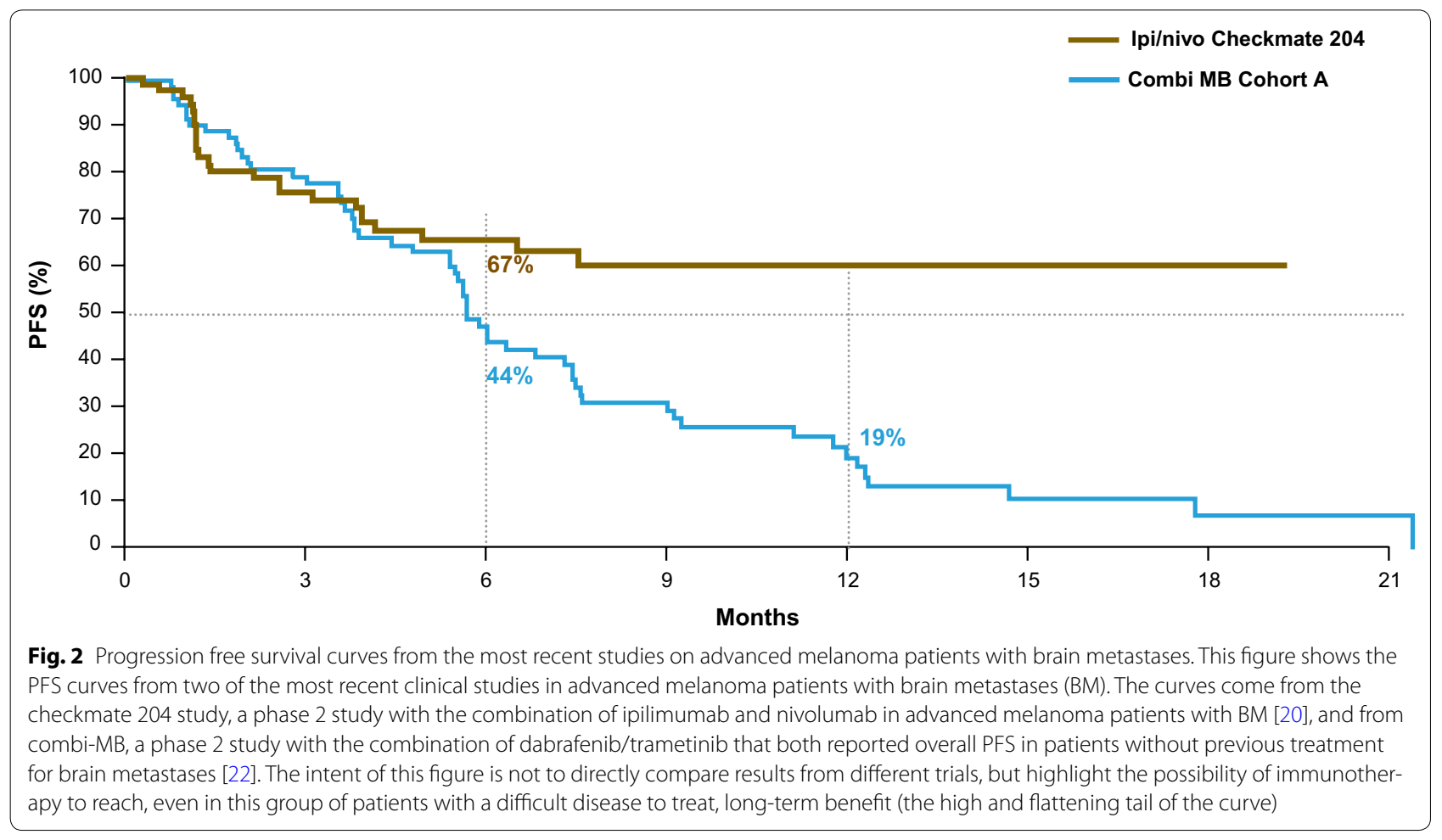




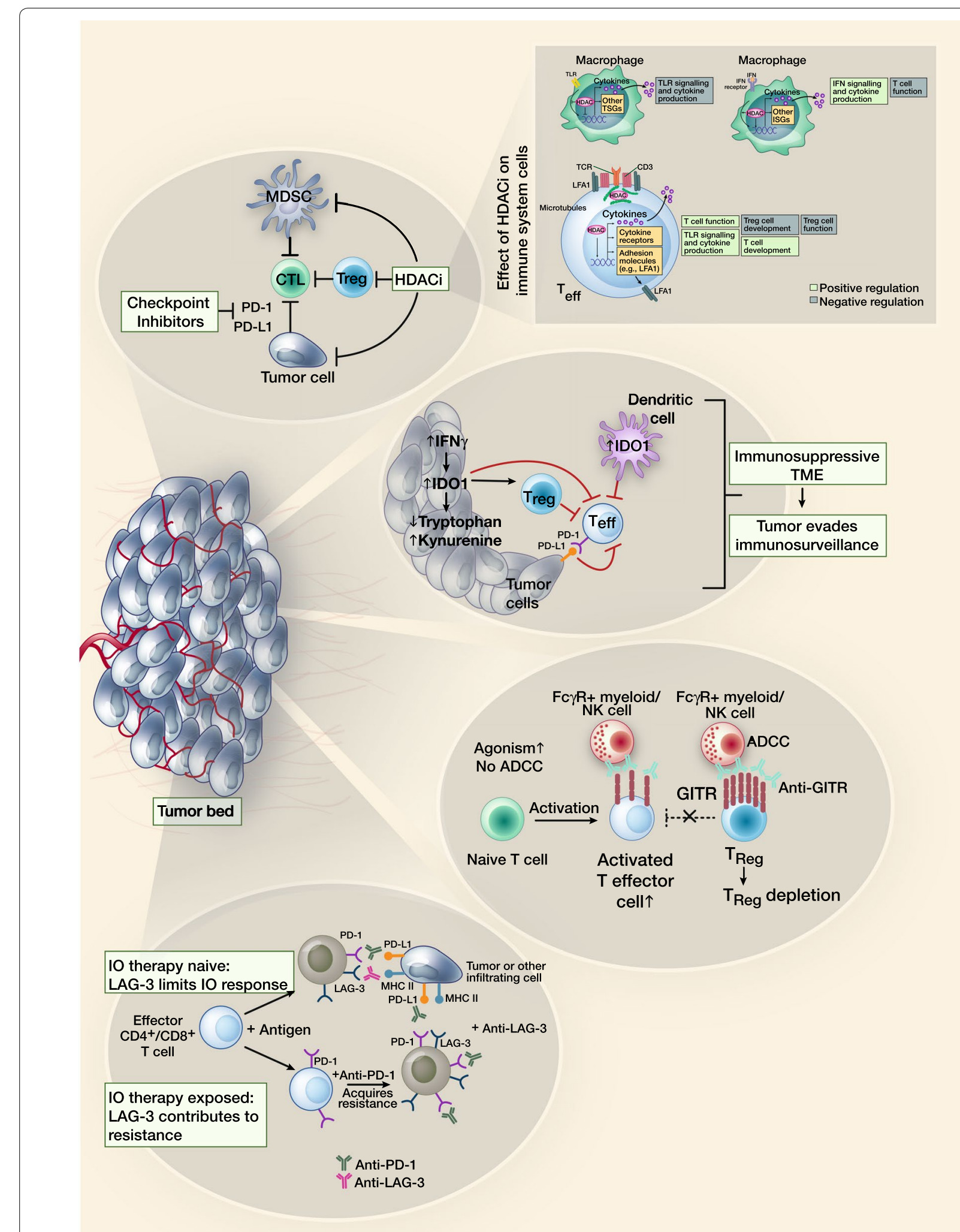

Fig. 3 New emerging pathways for future combination with anti-PD-1/PD-L1 compounds 
Epacadostat was investigated in combination with pembrolizumab, in the phase I/II ECHO-202/KEYNOTE-037 trial. In 38 patients with SCCHN, ORR was $34 \%$ and DCR was $61 \%$; ORR increased to $39 \%$ and DCR to $65 \%$ in 31 patients with only 1-2 lines of prior therapy [23]. Responses were observed regardless of PD-L1 expression or human papillomavirus (HPV) association. In the same trial, an ORR of $35 \%$ and DCR of $53 \%$ was observed In 40 patients with advanced urothelial carcinoma [24]. In this cohort, a higher response rate was observed in PD-L1-positive patients, although responses were still observed in PD-L1-negative patients. In 40 patients with NSCLC, ORR was $35 \%$ and DCR was $60 \%$ [25]. Epacadostat and pembrolizumab also showed clinical activity in patients with renal cell carcinoma $(\mathrm{n}=33)$ and $0-1$ prior lines of treatment, with an ORR of $47 \%$ and DCR of $58 \%$ [26]. In patients with advanced triple-negative breast cancer $(n=39)$ or ovarian cancer $(n=37)$, antitumour activity was similar to previously reported pembrolizumab monotherapy [27].

The combination was generally well tolerated across patient cohorts with a similar safety profile as pembrolizumab monotherapy, although there was a higher incidence of grade 3-4 rash [28]. Phase III studies of epacadostat plus pembrolizumab has completed accrual in melanoma and are planned in patients with SCHHN, NSCLC, urothelial carcinoma and renal cell carcinoma.

In the ECHO-204 study, patients with advanced solid tumors were treated with epacadostat (100 or $300 \mathrm{mg}$ twice-daily) in combination with nivolumab and included 40 patients with treatment-naïve advanced melanoma [29]. All six patients receiving epacadostat $100 \mathrm{mg}$ had a response while the ORR with the $300 \mathrm{mg}$ dose was $56 \%$ $(19 / 34)$. All responses were ongoing with a median duration of response of $16+$ weeks (range $<1+$ to $41+$ ) weeks.

Epacadostat plus nivolumab has also shown clinical activity and good tolerability in patients with other advanced solid tumours. As part of the ECHO 204 trial reported above, ORR was $23 \%$ and disease control rate (DCR) was $61 \%$ by modified Response Evaluation Criteria in Solid Tumors (mRECIST) in patients with previously-treated SCCHN. However, the combination with nivolumab did not demonstrate an efficacy signal in unselected populations of patients with refractory ovarian and colorectal cancer. Epacadostat plus nivolumab was generally well tolerated in patients with advanced solid tumours.

Another potential new treatment, entinostat, is a selective histone deacetylase (HDAC) inhibitor shown to enhance immune checkpoint inhibitor activity in preclinical studies. Preliminary data suggested that entinostat in combination with pembrolizumab showed promising activity in patients $(\mathrm{n}=13)$ refractory to previous treatment with checkpoint inhibitors [30]. However, toxicity was high with $62 \%$ of patients reporting treatmentrelated grade 3-4 adverse events.

Similarly, addition of the anti-lymphocyte activation gene-3 (anti-LAG-3) agent BMS-986016 to nivolumab showed encouraging initial efficacy and a similar safety profile to nivolumab monotherapy in 55 patients with advanced melanoma who had disease progression on or after anti-PD-1/PD-L1 therapy [31]. Sixty-seven percent of patients had M1c disease, 38\% had elevated lactate dehydrogenase (LDH) and 15\% had very elevated LDH, while $76 \%$ were heavily pretreated with more than two previous therapies and $40 \%$ had progressive disease as best response to previous anti-PD- 1 treatment. In this challenging cohort, ORR was $13 \%$, with a $20 \%$ response rate in patients with LAG- 3 expression $\geq 1 \%$ versus only $7 \%$ in LAG-3-negative $(<1 \%)$ patients. Expression of PD-L1 had no impact on response. Among six partial responses, three were in resistant patients and two in refractory patients. The safety profile was comparable to that of nivolumab monotherapy. These results are of particular interest in that, if the role of LAG-3 as a predictive biomarker is confirmed by future studies, this type of combination may be advantages over ipilimumab plus nivolumab or other combination regimens, allowing the personalization of immune-oncology based on the expression of different biomarkers.

Nivolumab has also been assessed in combination with the glucocorticoid-induced tumor necrosis factor receptor-related gene (GITR) agonist, BMS-986156 [32]. GITR is a costimulatory activating receptor that is upregulated upon T cell activation. Antitumour activity of GITR agonists may be via increased $T$ effector cell survival and function, reduced Treg-mediated suppression of $\mathrm{T}$ effector cells and Treg reduction through conversion to other immune cells. In a phase I/IIa study of in patients with advanced solid tumors, to date 29 patients have received BMS-986156 alone and 37 have received BMS-986156 plus nivolumab. No dose-limiting toxicities have been reported and the most common treatmentrelated adverse events have includes pyrexia, chills and fatigue, with all grade $1 / 2$ except in four patients. The safety profile of the combination was similar to monotherapy. The combination showed antitumor activity in several patients, increasing proliferating (Ki67+) NK and CD8 cells in peripheral blood and increasing the activation and proliferation of CD8 memory cells. Patients who responded included heavily pretreated patients and those who had previously progressed on anti-PD-1 therapy.

Finally, there is also interest in changing the tumour microenvironment to enhance immune cell localization and activation to overcome resistance to anti-PD-1/ PD-L1 therapies. These approaches include oncolytic 
viruses and small molecules such as toll-like receptor agonists [33] and STING-agonists. In a randomized phase II study involving 190 patients intratumoral injection of the oncolytic virus talimogene laherperepvec was combined with ipilimumab and compared with ipilimumab alone [34]. The ORR was $39 \%$ in patients receiving the combination therapy and $18 \%$ in patients receiving ipilimumab alone with acceptable toxicity in the combination arm. Future studies are required to define the activity of oncolytic viruses in combination with immune checkpoint inhibitors in patients progressing on anti-PD-1/PD-L1 treatment.

\section{Conclusions}

Data reported at ASCO 2017 has provided important new information regarding several of the outstanding questions that relate to I-O. These include new insights into possible mechanisms of resistance that might lead to new treatment combinations (e.g. anti-PD-1/PD-L1 agents with CD73 inhibitors) and the first data to show an ongoing benefit of 2 years pembrolizumab therapy almost a year after stopping treatment, with possible implications for the optimal duration of treatment with anti-PD-1 agents. More data has suggested a role for I-O in patients with melanoma brain metastases. In addition, new potential treatment combinations have been identified, including anti-PD-1 therapy in combination with an IDO inhibitor or a new anti-LAG-3 checkpoint inhibitor. Future years will hopefully see these questions more fully addressed as ongoing trials continue and new studies are initiated.

\section{Authors' contributions}

PAA and GAM drafted and approved the final manuscript. Both authors read and approved the final manuscript.

\section{Author details}

${ }^{1}$ Istituto Nazionale Tumori IRCCS Fondazione "G. Pascale", Via Mariano Semmola, 80131 Naples, Italy. ${ }^{2}$ Peter MacCallum Cancer Centre, Melbourne, VIC, Australia. ${ }^{3}$ University of Melbourne, Parkville, VIC, Australia.

\section{Acknowledgements}

Authors want to thank Nicole Jones for providing excellent support in realizing the figures, and Alessandra Trocino for providing excellent bibliography service and assistance. A special thanks to the Fondazione Melanoma Onlus for partially grant the present work.

\section{Competing interests}

Paolo A. Ascierto (PAA) has/had a consultant/advisory role for BMS, RocheGenentech, MSD, Array, Novartis, Amgen, Merck Serono, Pierre Fabre, Incyte. $\mathrm{He}$ also received research funds from BMS, Roche-Genentech, and Array. He is also Section Editor for Combination Strategies for Journal of Translational Medicine.

Grant A. McArthur (GAM) had research support from Celgene and Pfizer.

Availability of supporting data

Not applicable.

\section{Consent for publication}

Not applicable.
Ethical approval and consent to participate

Not applicable.

Funding

Not applicable.

\section{Publisher's Note}

Springer Nature remains neutral with regard to jurisdictional claims in published maps and institutional affiliations.

Received: 12 July 2017 Accepted: 1 August 2017

Published online: 08 August 2017

\section{References}

1. Eggermont AM, Chiarion-Sileni V, Grob JJ, Dummer R, Wolchok JD, Schmidt $\mathrm{H}$, et al. Prolonged survival in stage III melanoma with ipilimumab adjuvant therapy. N Engl J Med. 2016;375:1845-55.

2. Ascierto PA, Del Vecchio M, Robert C, Mackiewicz A, Chiarion-Sileni V, Arance A, et al. Ipilimumab 10 mg/kg versus ipilimumab $3 \mathrm{mg} / \mathrm{kg}$ in patients with unresectable or metastatic melanoma: a randomised, double-blind, multicentre, phase 3 trial. Lancet Oncol. 2017;18:611-22.

3. Goldberg SB, Gettinger SN, Mahajan A, Chiang AC, Herbst RS, Sznol M, et al. Pembrolizumab for patients with melanoma or non-small-cell lung cancer and untreated brain metastases: early analysis of a nonrandomised, open-label, phase 2 trial. Lancet Oncol. 2016;17:976-83.

4. Ribas A. Adaptive immune resistance: how cancer protects from immune attack. Cancer Discov. 2015:5:915-9.

5. Ribas A, Hamid O, Daud A, Hodi FS, Wolchok JD, Kefford R, et al. Association of pembrolizumab with tumor response and survival among patients with advanced melanoma. JAMA. 2016;315:1600-9.

6. Zaretsky JM, Garcia-Diaz A, Shin DS, Escuin-Ordinas H, Hugo W, HuLieskovan S, et al. Mutations associated with acquired resistance to PD-1 blockade in melanoma. N Engl J Med. 2016:375:819-29.

7. Gao J, Shi LZ, Zhao H, Chen J, Xiong L, He Q, et al. Loss of IFN- $\gamma$ pathway genes in tumor cells as a mechanism of resistance to anti-CTLA-4 therapy. Cell. 2016;167:397-404.

8. Ascierto PA, Simeone E, Sileni VC, Pigozzo J, Maio M, Altomonte M, et al. Clinical experience with ipilimumab $3 \mathrm{mg} / \mathrm{kg}$ : real-world efficacy and safety data from an expanded access programme cohort. J Transl Med. 2014:12:116.

9. Larkin J, Chiarion-Sileni V, Gonzalez R, Rutkowski P, Grob J-J, Cowey CL, et al. Overall survival results from a phase III trial of nivolumab combined with ipilimumab in treatment-naïve patients with advanced melanoma (CheckMate-067). In: Proceedings from the 2017 american association for cancer research Annual Meeting. Washington, DC; 2017 (abstract CT075).

10. Borghaei H, Paz-Ares L, Horn L, Spigel DR, Steins M, Ready NE, et al. Nivolumab versus docetaxel in advanced nonsquamous non-small-cell lung cancer. N Engl J Med. 2015;373:1627-39.

11. Fehrenbacher L, Spira A, Ballinger M, Kowanetz M, Vansteenkiste J, Mazieres J, et al. Atezolizumab versus docetaxel for patients with previously treated non-small-cell lung cancer (POPLAR): a multicentre, openlabel, phase 2 randomised controlled trial. Lancet. 2016;387:1837-46.

12. Herbst RS, Baas P, Kim DW, Felip E, Pérez-Gracia JL, Han JY, et al. Pembrolizumab versus docetaxel for previously treated, PD-L1-positive, advanced non-small-cell lung cancer (KEYNOTE-010): a randomised controlled trial. Lancet. 2016;387:1540-50.

13. Higgs BW, Morehouse CA, Streicher K, Brohawn PZ, Steele K, Rebelatto M, et al. A baseline IFNG gene expression signature correlates with clinical outcomes in durvalumab-treated advanced NSCLC cancer patients. In: Proceedings AACR Annual Meeting 2017. Washington, DC; 2017 (Abstr. 1773).

14. Streicher K, Higgs BW, Wu S, Coffman K, Damera G, Durham N, et al. Increased CD73 and reduced IFNG signature expression in relation to response rates to anti-PD-1(L1) therapies in EGFR-mutant NSCLC. J Clin Oncol. 2017;35 (suppl: abstr 11505) 
15. Martin P, Spitzmueller A, Wu S, Widmaier M, Korn R, Althammer S, et al. Mutually exclusive expression of CD73 and PDL1 in tumors from patients (pt) with NSCLC, gastroesophageal (GE) and urothelial bladder carcinoma (UBC). J Clin Oncol. 2017;35 (suppl: abstr 3079).

16. Young A, Ngiow SF, Madore J, Reinhardt J, Landsberg J, Chitsazan A, et al. Targeting adenosine in BRAF-mutant melanoma reduces tumor growth and metastasis. Cancer Res. 2017. doi:10.1158/0008-5472.CAN-17-0393.

17. Schadendorf D, Hodi FS, Robert C, Weber JS, Margolin K, Hamid O, et al. Pooled analysis of long-term survival data from phase II and phase III trials of ipilimumab in unresectable or metastatic melanoma. J Clin Oncol. 2015:33:1889-94.

18. Robert C, Ribas A, Hamid O, Daud A, Wolchok JD, Joshua AM, et al. Threeyear overall survival for patients with advanced melanoma treated with pembrolizumab in KEYNOTE-001. J Clin Oncol. 2016;34 (suppl: abstr 9503).

19. Robert C, Long GV, Schachter J, Arance A, Grob JJ, Mortier L, et al. Longterm outcomes in patients (pts) with ipilimumab (ipi)-naive advanced melanoma in the phase 3 KEYNOTE-006 study who completed pembrolizumab (pembro) treatment. J Clin Oncol. 2017;35 (suppl: abstr 9504).

20. Tawbi HA-H, Forsyth PAJ, Algazi AP, Hamid O, Hodi FS, Moschos SJ, et al. Efficacy and safety of nivolumab (NIVO) plus ipilimumab (IPI) in patients with melanoma (MEL) metastatic to the brain: results of the phase II study CheckMate 204. J Clin Oncol. 2017;35 (suppl: abstr 9507).

21. Long GV, Atkinson V, Menzies AM, Lo S, Guminski A, Brown MP, et al. A randomized phase II study of nivolumab or nivolumab combined with ipilimumab in patients (pts) with melanoma brain metastases (mets): the anti-PD1 brain collaboration (ABC). J Clin Oncol. 2017;35 (suppl: abstr 9508).

22. Davies MA, Robert C, Long GV, Grob JJ, Flaherty KT, Arance A, et al. COMBI-MB: a phase II study of combination dabrafenib (D) and trametinib ( $\mathrm{T}$ ) in patients (pts) with BRAF V600-mutant (mut) melanoma brain metastases (MBM). J Clin Oncol. 2017;35 (suppl: abstr 9506).

23. Hamid O, Bauer TM, Spira Al, Olszanski AJ, Patel SP, Wasser JS, et al. Epacadostat plus pembrolizumab in patients with SCCHN: preliminary phase I/II results from ECHO-202/KEYNOTE-037. J Clin Oncol. 2017;35 (suppl: abstr 6010)

24. Smith DC, Gajewski T, Hamid O, Wasser JS, Olszanski AJ, Patel SP, et al. Epacadostat plus pembrolizumab in patients with advanced urothelial carcinoma: preliminary phase I/II results of ECHO-202/KEYNOTE-037. J Clin Oncol. 2017;35 (suppl: abstr 4503)

25. Gangadhar TC, Schneider BJ, Bauer TM, Wasser JS, Spira AI, Patel SP, et al. Efficacy and safety of epacadostat plus pembrolizumab treatment of
NSCLC: preliminary phase I/II results of ECHO-202/KEYNOTE-037. J Clin Oncol. 2017;35 (suppl: abstr 9014)

26. Lara P, Bauer TM, Hamid O, Smith DC, Gajewski T, Gangadhar TC, et al. Epacadostat plus pembrolizumab in patients with advanced RCC: Preliminary phase I/II results from ECHO-202/KEYNOTE-037. J Clin Oncol. 2017;35 (suppl: abstr 4515)

27. Spira Al, Hamid O, Bauer TM, Borges VF, Wasser JS, Smith DC, et al. Efficacy/safety of epacadostat plus pembrolizumab in triple-negative breast cancer and ovarian cancer: phase I/II ECHO-202 study. J Clin Oncol. 2017;35 (suppl: abstr 1103)

28. Hamid O, Bauer TM, Spira Al, Smith DC, Olszanski AJ, Tarhini AA, et al. Safety of epacadostat $100 \mathrm{mg}$ bid plus pembrolizumab $200 \mathrm{mg}$ Q3W in advanced solid tumors: phase 2 data from ECHO-202/KEYNOTE-037. J Clin Oncol. 2017;35 (suppl: abstr 3012).

29. Perez RP, Riese MJ, Lewis KD, Saleh MN, Daud A, Berlin J, et al. Epacadostat plus nivolumab in patients with advanced solid tumors: preliminary phase I/II results of ECHO-204. J Clin Oncol. 2017;35 (suppl: abstr 3003)

30. Johnson ML, Gonzalez R, Opyrchal M, Gabrilovich D, Ordentlich P, Brouwer S, et al. ENCORE 601: a phase II study of entinostat (ENT) in combination with pembrolizumab (PEMBRO) in patients with melanoma. J Clin Oncol. 2017;35 (suppl: abstr 9529).

31. Ascierto PA, Melero I, Bhatia S, Bono P, Sanborn RE, Lipet EJ, al. Initial efficacy of anti-lymphocyte activation gene-3 (anti-LAG-3; BMS-986016) in combination with nivolumab (nivo) in pts with melanoma (MEL) previously treated with anti-PD-1/PD-L1 therapy. J Clin Oncol. 2017;35 (suppl: abstr 9520).

32. Siu LL, Steeghs N, Meniawy T, Joerger M, Spratlin JL, Rottey S, et al. Preliminary results of a phase I/lla study of BMS-986156 (glucocorticoidinduced tumor necrosis factor receptor-related gene [GITR] agonist), alone and in combination with nivolumab in pts with advanced solid tumors. J Clin Oncol. 2017;35 (suppl: abstr 104).

33. Leung ACF, Kummar S, Agarwala SS, Nemunaitis JJ, Gonzales R, Dabrick $J$, et al. Phase $1 \mathrm{~b} / 2$, open label, multicenter, study of intratumoral SD-101 in combination with pembrolizumab in anti-PD-1 naive and experienced metastatic melanoma patients. J Clin Oncol. 2017;35 (abstr 9550).

34. Chesney JA, Puzanov I, Ross MI, Collichio FA, Milhem MM, Chen L, Kim JJ, et al. Primary results from a randomized (1:1), open-label phase II study of talimogene laherparepvec (T) and ipilimumab (I) vs I alone in unresected stage IIIB- IV melanoma. J Clin Oncol. 2017;35:10 (abstr 9509).

\section{Submit your next manuscript to BioMed Central and we will help you at every step:}

- We accept pre-submission inquiries

- Our selector tool helps you to find the most relevant journal

- We provide round the clock customer support

- Convenient online submission

- Thorough peer review

- Inclusion in PubMed and all major indexing services

- Maximum visibility for your research

Submit your manuscript at www.biomedcentral.com/submit
(O) Biomed Central 